\title{
CONDIÇÕES DE SAÚDE BUCAL E DIABETES MELLITUS NA POPULAÇÃO NIPO-BRASILEIRA DE BAURU-SP
}

\author{
RELATION BETWEEN ORAL HEALTH CONDITIONS AND DIABETES \\ MELLITUS IN A JAPANESE POPULATION FROM BAURU-SP-BRAZIL
}

\author{
Nilce Emy TOMITA \\ Professora Doutora do Departamento de Odontopediatria, Ortodontia e Saúde Coletiva da Faculdade de Odontologia de Bauru-USP \\ Luiz Eduardo Montenegro CHINELLATO \\ Professor Doutor do Departamento de Estomatologia da Faculdade de Odontologia de Bauru-USP

\section{Laércio Joel FRANCO} \\ Professor Titular do Departamento de Medicina Social da Faculdade de Medicina de Ribeirão Preto-USP

\section{Magid IUNES} \\ Professor Titular do Departamento de Medicina Preventiva da Escola Paulista de Medicina-UNIFESP \\ José Alberto de Souza FREITAS \\ Superintendente do Hospital de Reabilitação de Anomalias Crânio-Faciais-USP

\section{Eymar Sampaio LOPES} \\ Professor Titular do Departamento de Odontopediatria, Ortodontia e Saúde Coletiva da Faculdade de Odontologia de Bauru-USP

\section{Grupo de Estudo de Diabetes em Nipo Brasileiros} \\ Alcides Hirai, Amélia T. Hirai, Helena Hariama, Laércio Joel Franco, Luiza Matsumura, Magid Iunes, Marly Cardoso Augusto, Mário \\ Kikuchi, Newton de Barros Jr., Nilce Tomita, Katsumi Osiro, Regina Moisés, Rita Chaim, Sandra Roberta Gouvea Ferreira, Sueli Godoy \\ Agostinho Gimeno e Katsunori Wakisaka.
}

E ste estudo transversal foi delineado para estimar a prevalência de diabetes mellitus, intolerância à glicose e condições de saúde bucal na população de origem japonesa, na faixa de 40 a 79 anos de idade, residente no município de Bauru-Brasil. Todos os indivíduos da primeira geração (isseis) e uma amostra casualizada de um terço da segunda geração (niseis) foram submetidos a entrevista domiciliar, totalizando 530 indivíduos. O exame clínico e teste oral de tolerância à glicose, e exame das condições de saúde bucal, foram realizados no Hospital de Reabilitação de Anomalias Crânio-Faciais - USP. Os dados foram processados através do programa Epi-Info, sendo que 22,9\% apresentaram ocorrência de diabetes mellitus (grupo I), 15,1\% tolerância à glicose diminuída (grupo II) e 61,9\% foram considerados normoglicêmicos (grupo III). O percentual de indivíduos desdentados totais foi de $45,9 \%$ para a amostra e valores de $58,4 \%, 46,7 \%$ e $41,2 \%$ foram verificados para os grupos I, II e III, respectivamente. Entre os indivíduos edêntulos, nenhum apresentava necessidade de confecção de prótese total, em contraposição aos dados encontrados para a população brasileira em levantamento realizado pelo Ministério da Saúde (1986), cuja necessidade era da ordem de 8,3\% na faixa etária de 35 a 59 anos. Pôde-se concluir que o edentulismo apresenta associação significante com a ocorrência de diabetes mellitus, porém não com a intolerância à glicose.

UNITERMOS: Diabetes mellitus; Saúde bucal; Epidemiologia; População nipo-brasileira. 


\section{INTRODUÇÃO}

A influência de diferenças geográficas e étnicas na ocorrência do diabetes mellitus tem sido amplamente estudada. O diabetes mellitus tipo 2 apresenta importante componente genético, com alta concordância em gêmeos idênticos ${ }^{4}$ e possui também muitos fatores ambientais em sua etiopatogenia ${ }^{2,23}$.

A população de origem japonesa constitui um interessante modelo para o estudo do diabetes, apresentando, originariamente, baixa prevalência desta doença. Com as mudanças sócio-culturais decorrentes dos movimentos migratórios, em curto período de tempo esta população passou a apresentar alto risco para o diabetes. Nos Estados Unidos da América, inquéritos populacionais com grupos de origem japonesa (nikkei) mostram uma prevalência de diabetes não-insulino dependente três a quatro vezes maior que a observada no Japão ${ }^{13}$.

Assim, populações de origem japonesa podem apresentar um risco inerente de expressar este tipo de diabetes com a "ocidentalização" ${ }^{2}$.

Alguns dados recentes do Japão mostram que a prevalência de diabetes está aumentando na última década, provavelmente refletindo mudanças no estilo de vida rumo a uma sociedade mais ocidentalizada ${ }^{4}$.

O movimento migratório para o Brasil iniciou-se em 1908, possibilitando a coexistência no país de diferentes gerações da população de origem japonesa. A maior população nikkei fora do Japão é encontrada no Brasil, com cerca de 70\% no estado de São Paulo ${ }^{6}$.

Alguns estudos demonstram evidências que o diabetes seja um importante problema de saúde nas populações nikkeis presentes no Brasil, apresentando coeficientes ajustados por idade duas vezes superiores àqueles encontrados no Japão ${ }^{14}$.

O Estudo Multicêntrico sobre a Prevalência de Diabetes Mellitus (DM) no Brasil, realizado pelo Ministério da Saúde a partir de 1986, aponta uma prevalência de DM de aproximadamente 7,5\% para a população adulta brasileira de ambos os sexos, na faixa de 30-69 anos ${ }^{11}$.

Umino; Nagao ${ }^{22}$, ao examinar 1012 pacientes de ambos os sexos, na faixa etária de 65 a 99 anos, atendidos na clínica gerontológica do Tokyo Medical and Dental University Hospital, verificaram que 7,6\% apresentavam diabetes mellitus.

Em face do significado epidemiológico desta enfermidade crônica em populações adultas, bem como dos expressivos números de adultos e idosos edêntulos em nosso meio, este estudo teve por objetivos avaliar as condições de saúde bucal da população de origem japonesa de Bauru-SP-Brasil quanto à história de cárie e perdas dentárias; e relacionar as perdas dentárias com a ocorrência de alterações na tolerância à glicose.

\section{MATERIAL E MÉTODOS}

$\mathrm{O}$ estudo foi desenvolvido em duas etapas. $\mathrm{Na}$ primeira, foi realizado um censo da população de ascendência japonesa residente em Bauru-SP-Brasil quanto à composição etária, sexo e geração, bem como quanto à presença de diabetes auto-referido, no ano de 1992.

A população identificada é mostrada na Tabela 1 , segundo o sexo e geração e na Tabela 2 segundo a geração e idade. Os dados indicam que o grupo de segunda geração (nisei) é o mais numeroso e o de primeira geração (issei) o mais idoso.

Este estudo transversal envolveu uma visita domiciliar e exames médico-odontológico e laboratorial em todos os indivíduos de primeira geração, com idades entre 40-79 anos e uma amostra aleatória de um terço (com um adicional de 20\%) de indivíduos de segunda geração, na mesma faixa etária, totalizando 530 indivíduos.

Todos os indivíduos da amostra foram submetidos aos seguintes procedimentos: entrevista domiciliar abordando questões demográficas e sociais, história médica e levantamento nutricional; exame médico (antropometria, pressão sangüínea, exame físico, Doppler vascular, eletrocardiograma e fundoscopia); exame laboratorial (testes de tolerância à glicose, lipídeos séricos e creatinina) e exame das condições de saúde bucal.

O exame clínico, teste oral de tolerância à glicose e exame das condições bucais foram realizados no Hospital de Reabilitação de Anomalias Crânio-FaciaisUSP, no período de maio a novembro de 1993.

O exame das condições de saúde bucal consistiu

TABELA 1- Distribuição da população nikkei de Bauru-SP segundo sexo e geração, 1992

\begin{tabular}{llccc}
\hline & \multicolumn{2}{c}{ Masculino } & \multicolumn{2}{c}{ Feminino } \\
\hline GERAÇÃo & $\mathbf{n}$ & $\%$ & $\mathbf{n}$ & $\%$ \\
\hline Issei & 186 & 13,5 & 178 & 11,3 \\
Nisei & 551 & 40,0 & 606 & 38,3 \\
Sansei & 410 & 29,8 & 467 & 29,6 \\
Mestiços & 228 & 16,5 & 329 & 20,8 \\
Total & 1375 & 100,0 & 1580 & 100,0 \\
\hline
\end{tabular}


de inspeção clínica visual com auxílio de espelho bucal plano e espátula de madeira, utilizando o índice CPO$\mathrm{D}^{7,14}$ para avaliar a história de cárie dentária neste grupo. Devido ao elevado índice de cárie para o grupo como um todo e ao grande número de indivíduos que apresentavam ausência total de dentes, no presente estudo a avaliação toma por base a dicotomia presença/ ausência total de dentes na boca.

Foi realizada a avaliação da condição das próteses dentárias por intermédio de inspeção visual, utilizando como critérios a adaptabilidade, desgaste e higiene da prótese.

\section{RESULTADOS}

A partir do censo realizado, foram identificados 339 indivíduos isseis entre 40-79 anos, dos quais 101 não participaram do presente estudo, devido a 40 recusas, 6 faltas ao exame e 55 mudanças/óbitos/não localização. Da amostra aleatória de 405 indivíduos niseis nesta faixa etária, houve 113 não participantes, dos quais 40 foram devido a recusas, 6 perderam o exame médico e 52 mudaram de endereço ou não foram localizados.
Observa-se que, nos últimos anos, tem ocorrido um forte movimento migratório de nipo-brasileiros para desenvolver trabalhos temporários no Japão e este fenômeno pode ter contribuído para o elevado número de indivíduos não participantes classificados como mudados ou não localizados.

A prevalência de diabetes mellitus e intolerância à glicose é apresentada na Tabela 3, por sexo e geração. Como o grupo de indivíduos isseis apresenta tendência a ser mais idoso que os niseis, pode-se notar facilmente a influência da idade ao comparar as taxas brutas e ajustadas por idade entre as gerações.

Os dados foram processados através do programa Epi-Info ${ }^{8}$, e pôde-se observar que 122 (22,9\%) indivíduos examinados apresentaram diabetes mellitus (grupo I), 80 (15,1\%) tolerância à glicose diminuída (grupo II) e 328 (61,9\%) foram considerados normoglicêmicos (grupo III) [Figura 1] ${ }^{12}$.

O percentual de indivíduos desdentados totais foi de $45,9 \%$ para a amostra [Figura 2] e valores de $58,4 \%$, $46,7 \%$ e $41,2 \%$ foram verificados para os grupos I, II e III, respectivamente [Figura 3]. O grupo I contava com 122 indivíduos, sendo que, destes, 71 (58,4\%) eram desdentados totais e $51(41,6 \%)$ possuíam dentes nos arcos dentários.

TABELA 2- Distribuição da população nikkei de Bauru-SP segundo geração e idade, 1992

IDADE (anos) GERAÇÃO (\%)

\begin{tabular}{lcccc}
\hline & $\begin{array}{c}\text { Issei } \\
(\mathrm{n}=364)\end{array}$ & $\begin{array}{c}\text { Nisei } \\
(\mathrm{n}=1157)\end{array}$ & $\begin{array}{c}\text { Sansei } \\
(\mathrm{n}=877)\end{array}$ & $\begin{array}{c}\text { Mestiços } \\
(\mathrm{n}=557)\end{array}$ \\
\hline$<20$ & 186 & 13,5 & 178 & 11,3 \\
$20-39$ & 551 & 40,0 & 606 & 38,3 \\
$40-69$ & 410 & 29,8 & 467 & 29,6 \\
$70 \mathrm{e}+$ & 228 & 16,5 & 329 & 20,8 \\
\hline
\end{tabular}

TABELA 3- Prevalência (\%) de diabetes mellitus e tolerância à glicose diminuída (TGD) na população nikkei de Bauru-SP, segundo sexo e geração, 1993

\begin{tabular}{|c|c|c|c|c|}
\hline \multicolumn{3}{|c|}{ Diabetes } & \multicolumn{2}{|c|}{ TGD } \\
\hline GERAÇÃO & Masculino & Feminino & Masculino & Feminino \\
\hline \multicolumn{5}{|l|}{ Issei } \\
\hline - Bruto & 22,8 & 15,3 & 14,9 & 19,8 \\
\hline - Ajustado-idade & 12,4 & 11,6 & 8,5 & 27,3 \\
\hline \multicolumn{5}{|l|}{ Nisei } \\
\hline - Bruto & 20,7 & 10,9 & 16,3 & 17,9 \\
\hline - Ajustado-idade & 21,7 & 11,4 & 19,3 & 17,0 \\
\hline
\end{tabular}




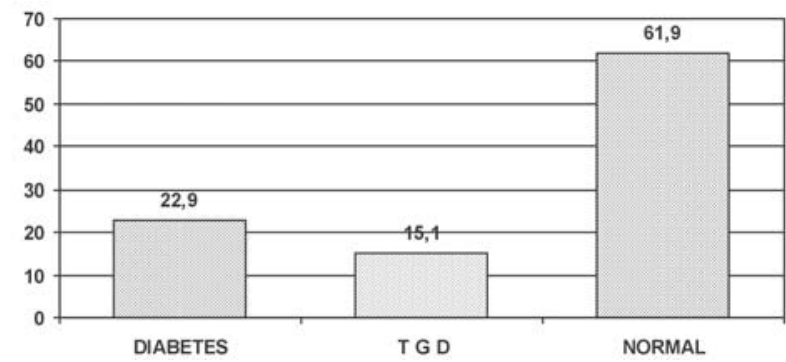

FIGURA 1- Distribuição da amostra entre indivíduos portadores de diabetes mellitus, tolerância à glicose diminuída (TGD) e normoglicêmicos. Bauru-SP, 1993

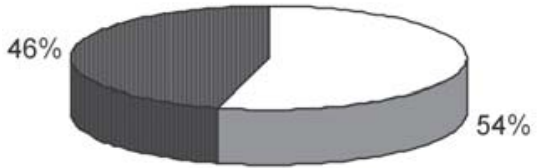

FIGURA 2- Distribuição da amostra segundo a presença de dentes (edentado) ou perda total de dentes (desdentado). Bauru-SP, 1993

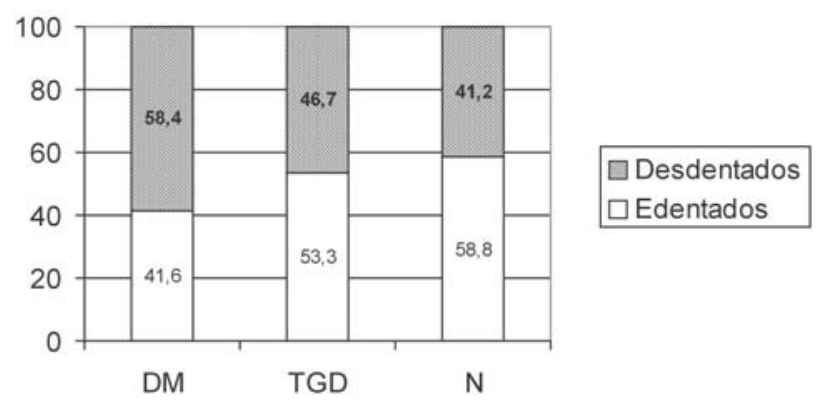

FIGURA 3- Distribuição percentual de desdentados e edentados entre pacientes portadores de diabetes mellitus (DM), tolerância à glicose diminuída (TGD) e normoglicêmicos (N). Bauru-SP, 1993

Aplicando-se o teste do qui-quadrado, comprovase uma associação estatisticamente significante quando comparados o grupo III contra o grupo I $\left(x^{2}=11,22\right.$; $\mathrm{p}=0,0008$ ), provando existir um maior percentual de desdentados na população com diabetes mellitus $(58,4 \%)$ que sem esta morbidade (41,2\%). Já a comparação entre os grupos II (46,7\%) e III (41,2\%) não mostrou associação estatisticamente significante entre as perdas dentárias e a tolerância à glicose diminuída.

Nesta amostra, entre os indivíduos desdentados, nenhum apresentava necessidade clínica de confecção de prótese total, o que contrasta com os dados encontrados para a população brasileira em levantamento realizado pelo Ministério da Saúde ${ }^{5}$ em
1986, cuja necessidade de prótese total era da ordem de 8,3\% na faixa etária de 35 a 59 anos.

\section{DISCUSSÃO}

Yabrudi ${ }^{24}$ considera que "a associação do diabetes mellitus com distúrbios bucais tem sido reconhecida, embora a mais freqüente e grave manifestação oral seja a doença periodontal”.

Ao comparar a saúde periodontal de adultos diabéticos e não diabéticos, Oliver; Tervonen ${ }^{16}$ verificaram que, enquanto perdas dentárias e de inserção gengival foram similares para ambos os grupos, diabéticos apresentaram maior prevalência e extensão de bolsas periodontais.

No presente estudo, foram observados maiores percentuais de perdas dentárias em diabéticos que nos grupos de pacientes intolerantes à glicose e não diabéticos, como decorrência provável de doença periodontal.

Observa-se, entre grupos populacionais adultos, um elevado percentual de desdentados. Neste grupo, a anamsese revelou que algumas particularidades culturais e a condição socioeconômica influenciaram a extração precoce de todos os dentes, com objetivo de instalar uma prótese total.

Com base nas informações fornecidas pelos indivíduos dos três grupos estudados, pôde-se inferir que uma parcela significativa dos dentes extraídos não apresentava indicação para tal procedimento cirúrgico. Assim, pode-se questionar se as perdas dentárias apresentadas por este segmento populacional guardam relação com a presença de doença periodontal ou cárie dentária.

Os achados de Bahru; $\mathrm{Abdu}^{3}$ mostraram alta prevalência de problemas dentais e periodontais entre pacientes diabéticos, em comparação aos observados em etíopes não diabéticos e são sugestivos das influências adversas do diabetes mellitus sobre a saúde bucal.

Sheppard ${ }^{19}$, no entanto, atribuiu a baixa ocorrência de cárie em seu estudo, como resultado da ausência de carboidratos na dieta e de uma dieta rica em vegetais. Rosa; Rocha; Lopes ${ }^{18}$ verificaram, em pacientes portadores de diabetes mellitus tipo I, de ambos os sexos e com idades entre 10 e 24 anos, que, apesar da restrição alimentar, os mesmos apresentaram a mesma suscetibilidade à cárie que os pacientes não diabéticos.

A questão alimentar reveste-se de importância ao analisar grupos que preservam valores culturais de seus países de origem. Os indivíduos isseis, nascidos no 
Japão, têm apresentado maior resistência ao processo de "ocidentalização". A dificuldade na aculturação reflete-se em uma alimentação essencialmente baseada em peixes, verduras e legumes, com baixo consumo de sacarose. Tais hábitos potencialmente poderiam apresentar influências positivas sobre a condição de saúde bucal desta população. Entretanto, o elevado número de desdentados nos três grupos, provavelmente, decorre da dificuldade de acesso à assistência odontológica, com a opção pela extração de todos os elementos dentais.

Ainda assim, é possível observar que, entre os indivíduos diabéticos, o percentual de desdentados é significantemente maior que entre os que apresentam intolerância à glicose e normalidade para esta condição, conforme a Figura $3(\mathrm{p}<0,001)$.

Albrecht; Bánóczy; Tamaz² observaram um menor número de lesões de cárie e mais dentes restaurados em pacientes diabéticos, atribuindo o fato a maiores cuidados com a saúde bucal. Em contraste, verificaram mais dentes perdidos, em função de doença periodontal, na faixa etária examinada, de 17 a 61 anos.

Em adultos, há relatos de maior prevalência de cárie para os indivíduos diabéticos em comparação aos não diabéticos ${ }^{2}$ quanto de prevalência semelhante entre ambos os grupos ${ }^{21}$. Alguns estudos apontam para uma relação negativa entre a prevalência de cárie dentária e o diabetes mellitus ${ }^{9,10}$. Outros estudos tendem a relacionar os níveis aumentados de glicose na saliva e sangue $^{9}$, a deficiência de vitamina $\mathrm{C}$ e complexo $\mathrm{B}^{9} \mathrm{e}$ a degeneração dos vasos ${ }^{20}$ em diabéticos com a presença de alterações na boca.

Levando em consideração os achados do presente estudo, observa-se que, apesar das dificuldades de coleta de informações em função do tempo decorrido desde a extração dos dentes e da impossibilidade de avaliar a real causa das perdas dentárias, houve um número significativamente maior de desdentados entre os portadores de diabetes mellitus que nos demais grupos.

Os resultados desta investigação ressaltam a importância de se desenvolver estudos prospectivos com esta poulação, particularizando a importância do controle metabólico do diabetes na freqüência e severidade de infecções bucais, como cárie e doença periodontal ${ }^{17}$.

\section{CONCLUSÕES}

A população nipo-brasileira de Bauru-SP apresenta uma elevada taxa de edentulismo (45,9\%), traduzindo uma baixa qualidade de saúde bucal.
Observou-se uma associação positiva entre a presença de diabetes e edentulismo. Há necessidade de se desenvolver estudos prospectivos nessa população, procurando conhecer a influência do grau de controle metabólico do diabetes na freqüência e severidade das infecções bucais. Por se tratar de uma população com elevada prevalência de diabetes mellitus, deve ser ressaltada a necessidade de intervenções que visem um bom controle metabólico do diabetes, bem como medidas direcionadas à sua prevenção, particularmente atuando na promoção de um estilo de vida saudável.

\section{AGRADECIMENTOS}

Aos acadêmicos da Faculdade de Odontologia de Bauru-USP Renato Hassunuma, Mônica Yamauti, Adriane Togashi, Miliza Miyaki, Edson Nakandakari, Mitsue Fujimaki, Celeste Okada e Denise Hosomi, pela participação na coleta de dados. À equipe do Departamento de Medicina Preventiva da Escola Paulista de Medicina-UNIFESP, pelas importantes contribuições. Ao Departamento de Odontopediatria, Ortodontia e Saúde Coletiva da FOB-USP e ao Hospital de Reabilitação de Anomalias Crânio-FaciaisUSP, pelo suporte operacional. Ao Prof. José Roberto Pereira Lauris, do Departamento de Odontopediatria, Ortodontia e Saúde Coletiva da FOB-USP, pela assessoria estatística.

\section{ABSTRACT}

The aim of this cross-sectional study was to determine the oral health condition in a Japanese population aged 40 to 79 , in Bauru, Brazil as well as its association with the occurrence of diabetes mellitus and impaired glucose tolerance. It involved 530 subjects, from both sexes. All persons of first generation (Issei) and a random sample of one third of second generation (Nisei) were submitted to a home interview. A clinical examination, oral glucose tolerance test, and examination of oral health conditions took place at the Hospital of Rehabilitation of Craniofacial Anomaly - USP. The data were processed by Epi-Info program and $22.9 \%$ of the individuals presented diabetes mellitus (group I), $15.1 \%$ impaired glucose tolerance (group II), and 61.9\% were considered normoglycemics (group III). The percentage of edentulous subjects was $45.9 \%$ for the total sample, and values of $58.4 \%, 46.7 \%$, and $41.2 \%$ were observed for groups I, II, and III, 
respectively. Among the edentulous subjects, no one showed necessity of making a total prothesis. These data indicate that tooth loss showed significant association with the occurrence of diabetes mellitus, but there was no significant association with glucose intolerance.

UNITERMS: Diabetes mellitus; Oral health; Epidemiology; Japanese population

\section{REFERÊNCIAS BIBLIOGRÁFICAS}

1- Akazawa Y. Prevalence and incidence of diabetes mellitus by WHO criteria. Diabetes Res Clin Pract 1994; 24 (Suppl):523-7.

2- Albrecht M, Bánóczy J, Tamáz Jr G. Dental and oral symptoms of diabetes mellitus. Community Dent Oral Epidemiol 1988; 16(6):378-80.

3- Bahru Y, Abdu SS. A study of dental problems in diabetic patients. Ethiop Med J; 1992; 30(2):95-103.

4- Barnett AH et al. Diabetes Mellitus in identical twins. A study of 200 pairs. Diabetologia 1991; 20:87-93.

5- Brasil. Ministério da Saúde. Divisão Nacional de Saúde Bucal. Levantamento Epidemiológico em Saúde Bucal - Brasil, Zona Urbana, 1986. Brasília : Centro de documentação do Ministério da Saúde, 1988.

6- Centro de Estudos Nipo-Brasileiros. Pesquisa da população de japoneses residentes no Brasil. São Paulo : Centro de Estudos Nipo-Brasileiros; 1988.

7- Chaves MM. Odontologia social. 3.ed. Rio de Janeiro : Artes Médicas; 1986.

8- Dean AG, Dean JA, Burton AH, Dicker RC. Epi-info version 5: a word processing, database, and statistics program for epidemiology on micro-computers. Atlanta : Centers for Disease Control; 1990

9- Davina AB. La diabetes en odontologia. An Esp Odontol 1974; 33(1):59-68.

10- Faulconbridge AR, Bradshaw WC, Jenkins PA, Baum JD . The dental status of a group of diabetic children. Brit Dent J $1981 ; 151: 253-5$

11- Franco LJ, Malerbi DA. Prevalence of diabetes mellitus and impaired glucose tolerance in Brazilian population aged 40-69 yr [Abstract]. Diabetes 1991; 40(Suppl.1):304 A.

12 - Franco LJ, Hirai A, Hirai AT, Tancuchi C, Osiro K, Wakisaka K, Souza Freitas JA de, Iochida LC, Marcopito LF, Matsumura LK, Iunes M, Kikuchi M, Tomita NE, Miyazaki N, Chain R. Diabetes in japanese-brazilians - influence of the Acculturation Process, 1995. Diabetes Res Clin Practice 1996; 34(Suppl): S517.
13- Fujimoto WY, Leonetti DL, Kinyoun JL, Newell-Morris L, Shuman WP, Stolov WC, Wahl PW. Prevalence of diabetes mellitus and impaired glucose tolerance among second generation Japanese American men. Diabetes 1987; 36:721-9.

14- Gotlieb SLD. Mortalidade de imigrantes japoneses residentes no município de São Paulo; 1988 [Tese de Doutorado - Faculdade de Saúde Pública da USP].

15- Klein H, Palmer. Dental caries in American indian children. Public Health Bull n.239, Wash. 1937. Govt. Printing office, 1938 apud Chaves $\mathrm{MM}^{7}$

16- Oliver RC, Tervonen T. Diabetes - a risk factor for periodontitis in adults? J Periodontol 1994; 65(5):530-8.

17- Rees TD. The diabetic dental patient. Dent Clin North Am 1994; 38(3):447-63.

18- Rosa OPS, Rocha RSS, Lopes ES. Imunoglobulinas salivares e prevalência de cárie em portadores de diabetes mellitus insulinodependente (DMID). Rev Fac. Odontol Bauru 1993; 1(1/4):1821.

19- Sheppard IM. Oral manifestations of diabetes mellitus. A study of one-hundred cases. J Amer Dent. Assoc 1942; 29(9):1188-92.

20- Stallard RE. Enfermidad periodontal en pacientes diabeticos. Inden 1981; 6(4):46-9.

21- Tenovuo J, Alanen P, Larjava H, Viikari J, Lehtonen OP. Oral health of patients with insulin-dependent diabetes mellitus. Scand J dent Res 1986; 94(4):338-46.

22- Umino M, Nagao M. Systemic diseases in elderly dental patients. Int Dent J 1993; 43:213-8.

23- World Health Organization. Diabetes Mellitus - Report of a WHO Study Group. Technical Report Series 727. Geneva, WHO, 1985.

24- Yabrudi MAL. Oliveira Filho JB de, Alves dos Santos R. Saúde bucal dos pacientes diabéticos. Rev Gaúcha Odontol, 1993; 41(2):105-9.

Correspondência para:

Prof.a Dra. Nilce Emy Tomita

Faculdade de Odontologia de Bauru-USP

Departamento de Odontopediatria,

Ortodontia e Saúde Coletiva

Al. Octávio Pinheiro Brisolla, 9-75

Cep.: 17012-901 - Bauru-SP

E-mail: netomita@usp.br 